\title{
Development of a Vibration Signal Acquisition and Analysis System Using LabVIEW
}

\author{
Dong $\mathrm{GUO}^{\mathrm{a}}$, Linzhang $\mathrm{JI}^{\mathrm{b}, \mathrm{1}}$ and Xianghua $\mathrm{MA}^{\mathrm{a}}$ \\ ${ }^{a}$ School of Electrical and Electronic Engineering, Shanghai Institute of Technology, \\ China \\ ${ }^{\mathrm{b}}$ School of Mechanical Engineering, Shanghai Institute of Technology, China
}

\begin{abstract}
In order to meet the requirements of engineering testing and reduce system development costs, LabVIEW 8.0 is used as the software development platform to design a set of equipment vibration signal acquisition and analysis system. The signal of the device under test is collected through the sensor, and then transferred to the LabVIEW development platform through the computer after hardware conditioning. In addition to displaying the waveform, the platform can realize time domain analysis and frequency domain analysis through system processing; the system also has data storage and offline signals Analysis and other functions. By comparing the turntable data of the vibration test bench measured by the system with the existing test data, it proves that the system is complete and accurate. At the same time, the system is applied to the on-site inspection of tamping coke oven equipment.
\end{abstract}

Keywords. LabVIEW, vibration signal, data acquisition, signal analysis.

\section{Introduction}

As an important fault diagnosis method, the acquisition and analysis of vibration signals are widely used in industry and engineering by virtue of the advantages of not disassembling the body, not damaging the body itself, not affecting the normal operation of the equipment, and wide measurement range [1]. There is a tamping coke oven equipment ' which machine body vibrates abnormally and accompanied by noise during the tamping and coking process, which affects the normal construction. It is necessary to perform vibration detection on the machine body and analyze the cause of the fault

${ }^{1}$ Corresponding Author, Linzhang JI, School of Mechanical Engineering, Shanghai Institute of Technology, China; E-mail: e-mail: jlz81@163.com. 
according to the fault characteristics. Both the machine body and the testing equipment are hard to move because of their huge body, therefore we need to find another method.

The vibration signal acquisition and analysis system includes signal acquisition and real-time analysis software and hardware, data management and signal processing software, and the acquisition unit can realize data acquisition of sinusoidal vibration, shock vibration, and random vibration of the equipment. The data analysis software can perform time-domain and frequency-domain analysis of the collected signals, such as eigenvalue extraction, spectrum analysis, order analysis, modal analysis, etc[2-4].

With the development of virtual instruments and the rapid development of automation technology, LabVIEW has gradually become a popular program development platform. As a graphical editing language [5], LabVIEW uses a graphical editing language that creates applications in the form of icons. It has the characteristics of intuitive graphics, easy to learn, general programming, and modularity compared with the text creation application programming language [6]. The vibration signal acquisition and analysis system designed by LabVIEW reduces software development costs and error rates, improves work efficiency, provides convenience for the realization of a friendly human-computer interaction interface [7], and beside it provides great convenience for vibration detection of tamping coke oven equipment.

\section{Overall Scheme of the System}

The acquisition and analysis system are composed of software and hardware [8]. The software uses LabVIEW 8.0 as the core part of the whole system to realize various waveform display and analysis and processing functions of the measured vibration signal [9]. It is composed of piezoelectric acceleration sensor, NI USB -4432 acquisition instrument and computer are used as hardware platforms to implement the acquisition, signal amplification, isolation and filtering of the measured signals.

\section{Hardware System Structure}

The system hardware is mainly composed of piezoelectric acceleration sensor, NI USB-4432 acquisition instrument, computer, data connection line and so on.

Piezoelectric acceleration sensor:As a spontaneous sensor, it converts the vibration signal into electrical signal output through the piezoelectric effect of the piezoelectric sheet, and the spring ball is connected to the substrate to reduce the resonance frequency. At the same time, the spring reduces the consumption of vibration energy, prolongs the vibration decay time, and enhances the vibration effect. In addition to light weight, small size, and easy installation, it also has the characteristics of large dynamic range, high accuracy, and long-term temperature stability.

NI USB-4432 acquisition instrument: This system uses the USB-4432 data acquisition instrument developed by NI. The acquisition instrument comes with signal conditioning functions, such as signal amplification, isolation, filtering, etc. It provides acceleration sensor signal conditioning with a dynamic range of $80 \mathrm{~dB}-100 \mathrm{~dB}$ and integrated software optional IEPE ( $2.1 \mathrm{~mA}$ constant current). The module has four analog input channels with a sampling rate range of $2-102.4 \mathrm{KS} / \mathrm{s}$ and a single analog output channel. The acquisition instrument is suitable in size, simple to connect, and easy to carry, making it an ideal portable test application. 


\section{Software System Structure}

The system software design part mainly includes modules such as data collection and display, signal storage and analysis, and historical record playback. The software system structure is shown in figure 1.

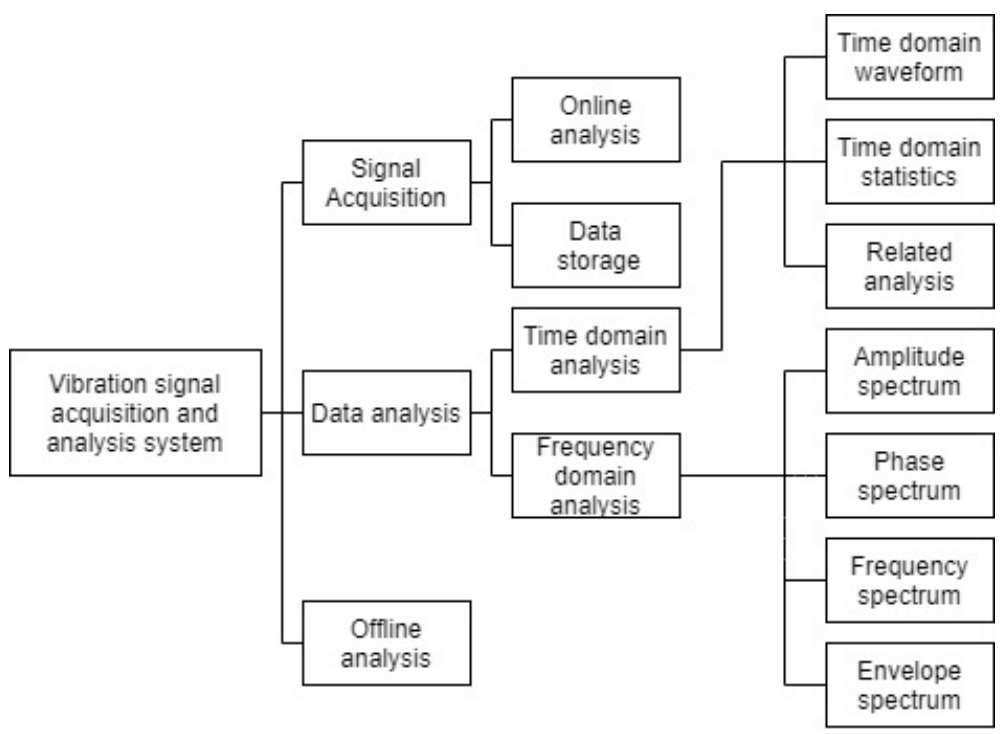

Figure 1. System software structure design drawing

\subsection{Data Acquisition Module}

The data acquisition module is one of the most important functions in LabVIEW, providing a data source for the entire system. The data acquisition module can pick up the vibration signal of the equipment and set the parameters accordingly, including the selection of the acquisition channel, the change of the signal connection mode and the setting of other related sampling information, so as to achieve continuous acquisition of the signals of multiple channels, to achieve real-time monitoring of equipment. The data acquisition function of this system is mainly composed of DAQmx create channel, DAQmx start task, DAQmx read task, DAQmx stop task, etc., and convert the vibration signal into an analog voltage signal type to realize the analog input function. The partial structure of the data acquisition module is shown in figure 2 below.

\subsection{Data Analysis Module}

As the most critical part of the vibration signal acquisition and analysis system, the data analysis module can not only monitor the equipment vibration signal in real time, but also obtain the characteristic parameters of the equipment vibration signal at multiple levels, and feedback to the user in a clearer way, so that the user can understand the equipment work Status, and deal with equipment failures in time.

In this system, the data analysis module can perform online analysis of real-time signals and offline analysis of historical data. Users can integrate the analysis function 
into a graphical module and achieve signal time domain analysis and spectrum analysis by click the corresponding icon.

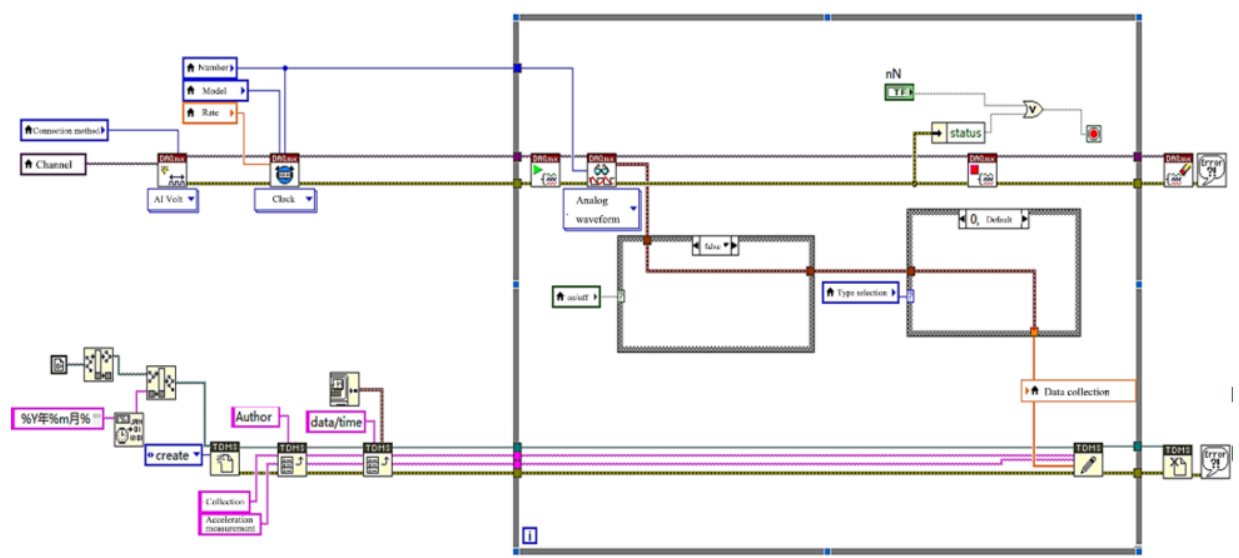

Figure 2. Part of the data collection program

Time domain analysis: It is an analysis method based on the relationship between signal amplitude and time. It obtains real-time characteristic parameters in the working status of the equipment and provides effective information for equipment dynamic analysis and fault diagnosis [10] Through time-domain statistical analysis and autocorrelation analysis of the equipment status signal.

Spectrum analysis: In the fault diagnosis, the frequency structure of the signal is often changed due to the occurrence of a fault. In order to more intuitively understand the operating status of the entire equipment and various components, mathematical processing methods are used to transform the time domain analysis of the signal into frequency domain analysis to perform spectrum analysis. The spectrum analysis in this system includes a variety of spectrum analysis such as power spectrum and amplitude spectrum [11].

Part of the program panel is shown in figure 3.

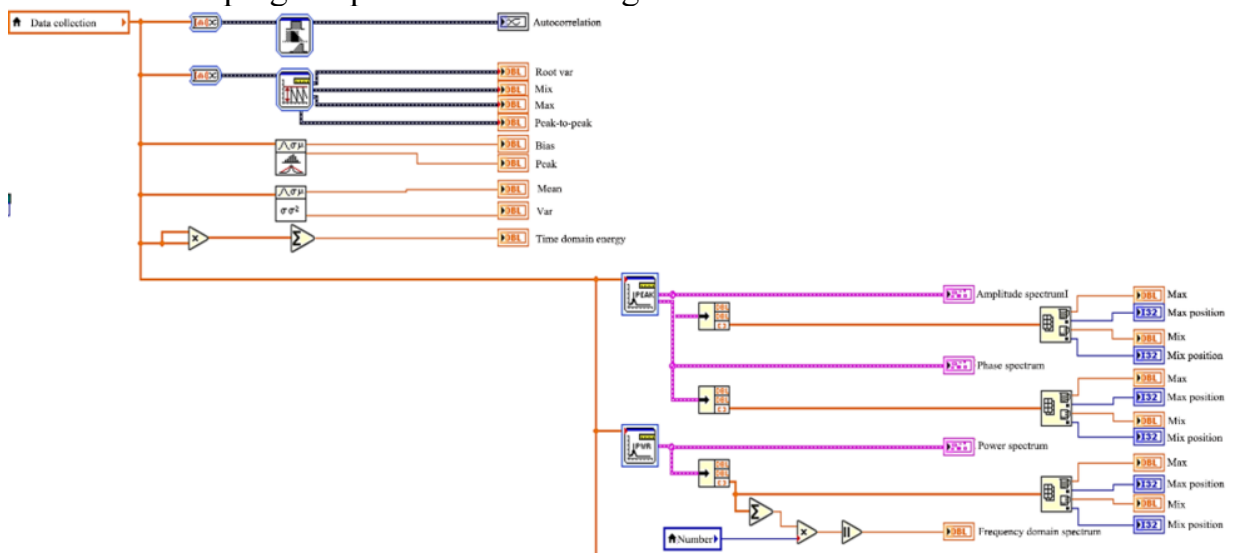

Figure 3. Part of the program panel 


\subsection{Data Storage and Query Module}

The data storage and query module realize it function through the Access database, which can not only realize the waveform storage function but also the historical data playback function. The user can store the vibration data in the Access database during the system test and store it in the "tdms" format. The "file name" and "save path" can be selected according to the needs.

In addition to the sampling data, the storage content also records related information such as sampling channel, sampling type, and sampling time, as shown in figure 4 below. In the data storage and query module, the user clicks the "Save" button, and the system automatically pops up a dialog box to display the storage status. After the storage is complete, the user can retrieve the data file in the corresponding folder, and view and analyze the original test data on the program front panel.

\begin{tabular}{|c|c|c|c|c|c|c|c|}
\hline Root Name & Title & Author & Date/Time & Groups & Description & & \\
\hline & & & & 1 & & & \\
\hline Groups & Channels & Description & & & & & \\
\hline Signal Acquisition & & & & & & & \\
\hline \multirow{2}{*}{\multicolumn{8}{|c|}{ Channel }} \\
\hline & & & & & & & \\
\hline Acceleration meas & Datatype & Unit & Lenth & Mininum & Maxinum & Description & date_time \\
\hline & DT_DOUBLE & & & & & & $2020 / 9 / 9$ \\
\hline
\end{tabular}

Figure 4. Data storage information

\section{System Applications}

\subsection{Test and Analysis of Eccentric Vibration of Turntable}

\subsubsection{Background}

The laboratory of the School of Mechanical Engineering has a test bench for vibration testing, and the turntable on the test platform has an eccentricity problem. In order to find out the cause of the eccentricity, the system is used to test and analyze the vibration caused by the eccentricity of the rotating shaft. Specifically, an acceleration sensor and NI USB-4432 acquisition instrument are used to collect vibration signals, and a signal acquisition and analysis system built by LabVIEW is used to process the vibration waveforms to obtain test data and compare them with the original data.

\subsubsection{System Connection}

This system is to collect and analyze the vibration signal of the turntable of the vibration test platform. The on-site connection of equipment and instruments is shown in figure 5 . Mainly include: School of Mechanical Engineering vibration test platform, NI USB-4432 acquisition instrument, acceleration sensor, computer. 


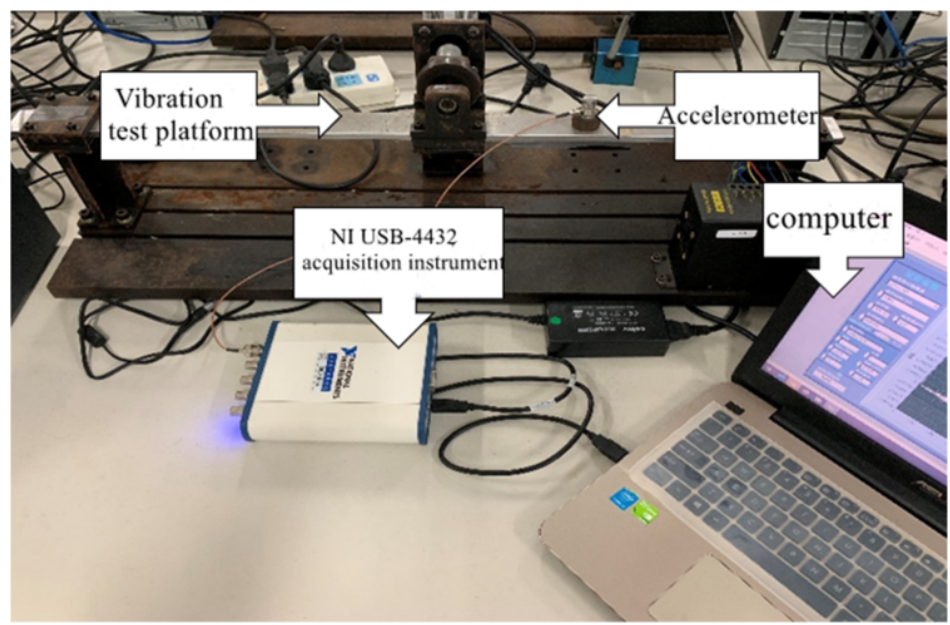

Figure5. Device hardware connection structure diagram

\subsubsection{Collection and Cause Analysis of Eccentric Vibration Signal of Rotating Shaft}

This application adopts the pseudo-differential signal connection method to reduce the influence of noise, sampling in continuous mode, and the sampling frequency is $1000 \mathrm{HZ}$. Filter by Butterworth filter, and use windowing to reduce spectral energy leakage. The $1 \mathrm{~s}-4 \mathrm{~s}$ waveform is shown in figure 6 . The time domain analysis of the group of vibration signals is performed, and the time domain index statistics [12] [13] of the vibration signals are shown in table 1.

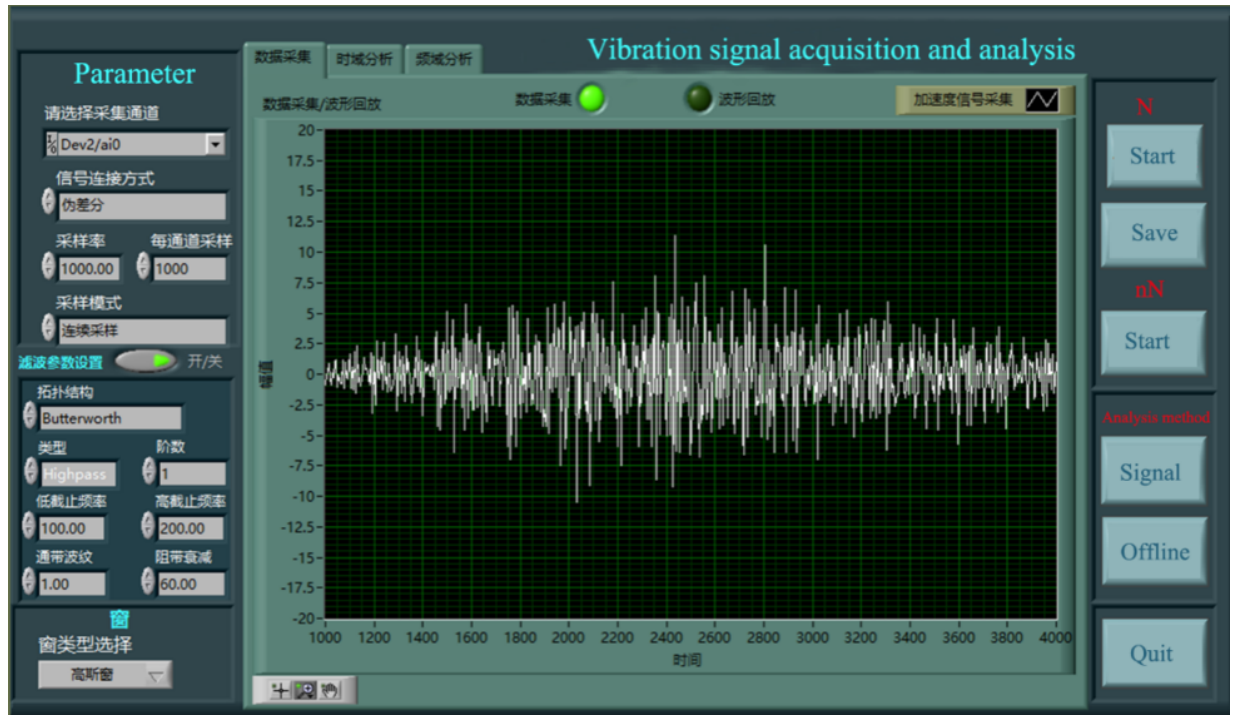

Figure 6. Vibration signal waveform 
Table 1. Time domain index statistics of vibration signal

\begin{tabular}{ccc}
\hline No. & Index & Acceleration \\
\hline 1 & Max & 10.9472 \\
2 & Mix & -10.0984 \\
3 & Mean & -1.15943 \\
4 & Mean square & 20.8487 \\
5 & Variance & 9.8666 \\
\hline
\end{tabular}

Through intercepting the waveform of this time period to spectrum analysis, we use the amplitude spectrum to reflect the linear distribution formed by the amplitude of each harmonic in the time domain signal with frequency, such as which of the vibration signals are harmonic components, which signals are fault components, etc. The rotating speed of the turntable of this test platform is 540r/min, and it has the characteristic of eccentricity. According to the frequency spectrum of the turntable with a frequency of $0-20 \mathrm{~Hz}$ in figure 7 , a peak appears when the rotating frequency is $9 \mathrm{~Hz}$, accompanied by $1 / 2$ frequency $(5 \mathrm{~Hz})$ and double frequency $(12 \mathrm{~Hz})$. Theoretically speaking, if the rotating equipment turntable has the characteristic of eccentricity, the amplitude of the rotation frequency of the axis where the eccentric equipment is located will increase, and at the same time there will be frequency doubling. In order to prove the reliability of the program, the data was compared with the data measured by traditional instruments at the same time, and the results were the same. Other analysis maps are not listed here.

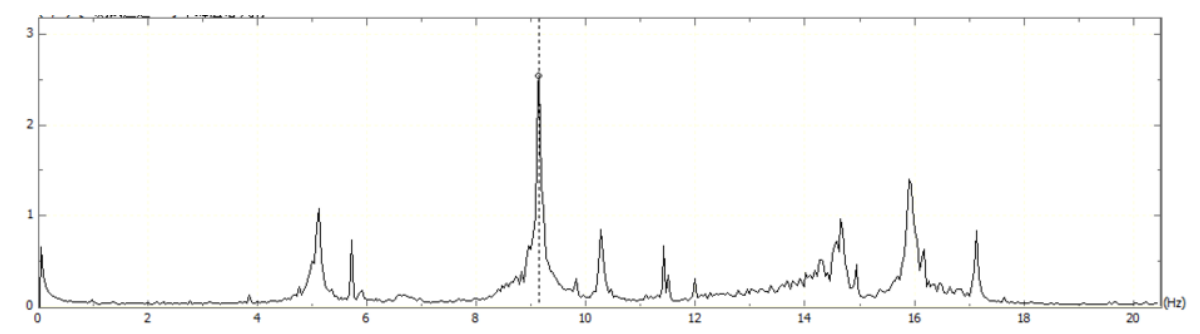

Figure 7. Turntable frequency spectrum of vibration test bench

\subsection{Vibration Detection and Analysis of a Coke Oven Tamping Machine}

\subsubsection{Background}

Increasing coke output, production efficiency, and reducing pollution are the core indicators of coke production for enterprises in the coal coking industry. Although many improvements and technical upgrades have been made on the basis of the introduction, digestion and absorption of tamping technology from German $\mathrm{KOCH}$, there is still a certain gap compared with developed countries. Among them, one of the main problems restricting the development of tamping coking technology is Stamping Charging Pushing (SCP for short), especially as the height of the coking chamber increases, The active load of tamping impact is correspondingly increased, and the impact of vibration during the tamping process will be more serious, including impacts on the stability of briquettes, the life of the mechanical, electrical, and hydraulic components of the equipment, the safety and reliability of the equipment, and the coke 
output, Production efficiency, etc. For this reason, the author of this article has carried out the vibration test analysis and structural optimization study of a domestic company's $6.25 \mathrm{~m}$ SCP integrated machine to improve market competitiveness. And apply this system in this project.

\subsubsection{Vibration Detection and Analysis of Coke Oven Tamping Machine}

As shown in figure 8, during the vibration detection process of the coke oven tamping machine, the piezoelectric acceleration sensor is fixed on the base support of the tamping coal charging pusher (SCP) integrated machine body, and the NI USB-4432 acquisition instrument Connect the sensor and the computer vibration detection system respectively, and test after setting the collection parameters.

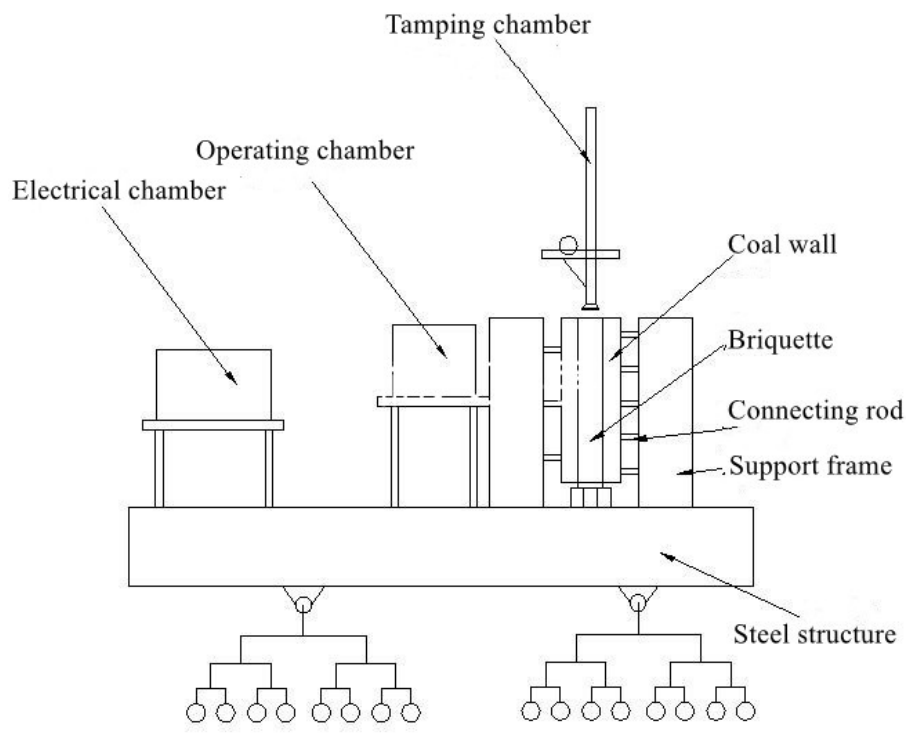

Figure 8. Schematic diagram of $6.25 \mathrm{~m}$ tamping coal charging pusher (SCP) integrated machine

Figure 9 shows the waveform of the vibration signal at 400s-450s. Perform time domain analysis on this group of vibration signals, and the time domain indicator statistics of vibration signals are shown in table 2. 


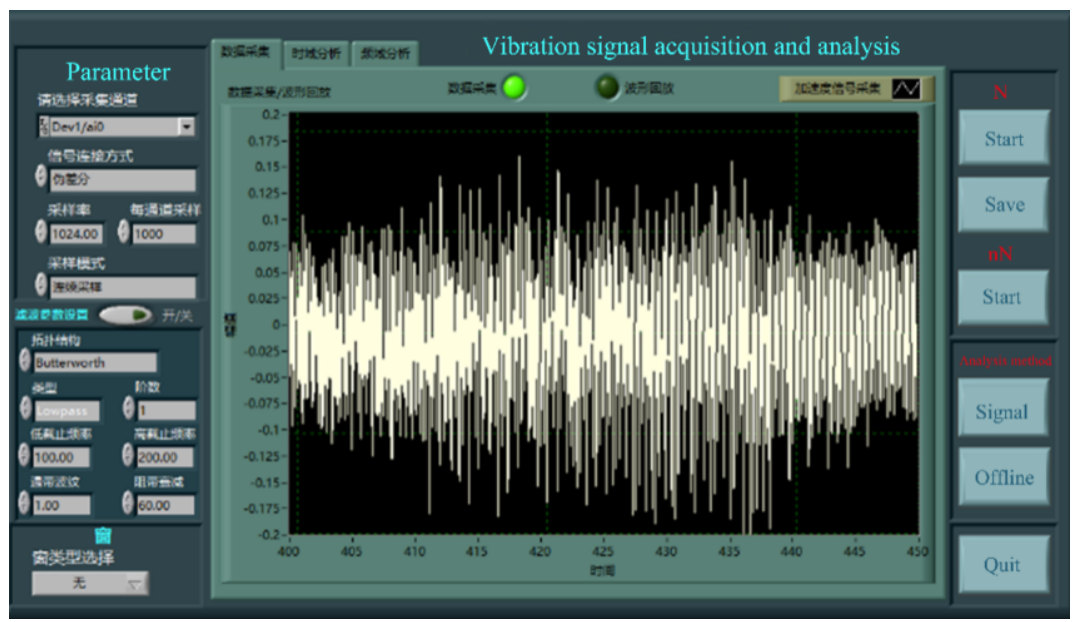

Figure 9. Vibration signal waveform diagram of coke oven tamping machine

Table 2. Time domain index statistics of vibration signal

\begin{tabular}{ccc}
\hline No. & Index & Acceleration \\
\hline 1 & Max & 63.2726 \\
2 & Mix & -76.0793 \\
3 & Mean & 0.0033 \\
4 & Mean square & 18.573 \\
5 & Variance & 344.957 \\
\hline
\end{tabular}

We get the self-spectrogram through intercepting the waveform of this time period for spectrum analysis, as shown in figure 10 . Among them, when the frequency is $8 \mathrm{~Hz}$, the peak value reaches $8.2522 \mathrm{~mm} / \mathrm{s}$.

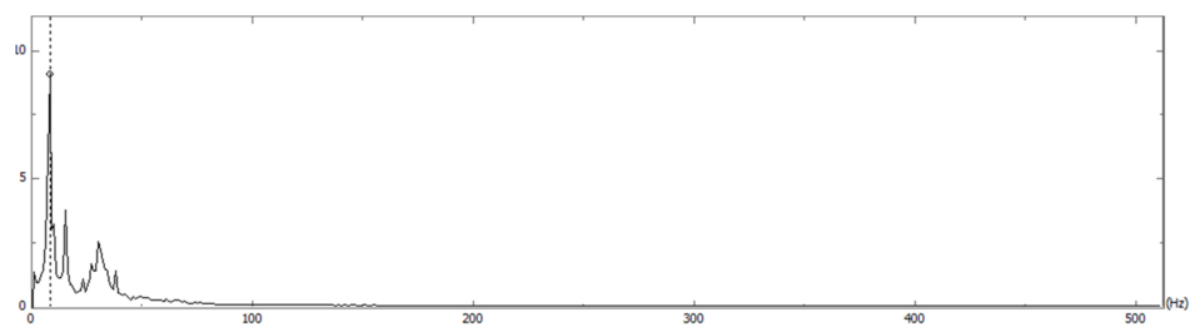

Figure 10. Frequency spectrum of coke oven tamping machine

\subsection{System Application Description}

The above two application cases illustrate: The designed system can not only display and process the collected real-time signals, but also replay and calculate the historical data measured by the system. Through the collection and analysis of equipment vibration signals, and the comparison with traditional testing equipment data, it is proved that the designed system is completely reliable and usable. 


\section{Conclusion}

In this paper, we develop an integrated system for vibration signal monitoring through LabVIEW, and establish a friendly human-computer interaction interface. After the vibration signal of the collected equipment is processed by LabVIEW, various functions such as time domain analysis and spectrum analysis are realized. Application shows: Compared with traditional monitoring instruments, the vibration signal acquisition and analysis system established by virtual instruments not only improves development efficiency and reduces development costs, but also improves work intensity and environment, and is more conducive to the design of friendly human-computer interaction interfaces. It is convenient for users to accurately judge and pre-process the equipment running status.

\section{Acknowledgments}

This work was supported by National Key R\&D Program of China (Grant:2020YFB2007700).

\section{References}

[1] Jin Xiaohang, Sun Yi, Shan Jihong, etal. Fault diagnosis and prognosis for wind turbines: An overview [J]. ChineseJournal of Scientific Instrument, 2017, 38(5) : 1041-1053.

[2] Ni Tongpan, Liang Wei. Research on Design and Application of Aircraft Electromechanical Integrated Management System Based on Model[J]. Aviation Science and Technology,2017,28(6):74-78.

[3] Zhang Xiangyan, Wang Tieyong, Research on Computer Fault Tolerant Technology Based on Airborne Electromechanical Control and Management[J]. Microelectronics and Computer,2008,25(12):125 - 127.

[4] Chen Fu, Wang Shanhu, Duan Haijun, etal. Research on Redundancy Management Technology of Aircraft Electromechanical Core Processor[J]. Information and Communication,2016(10):29-30.

[5] Huang Yunjiang. The realization of data acquisition based on LabVIEW[J]. Fujian Computer, 2008,24(4): 114,99 .

[6] Chen Pengfei, Li Luping, Zhang Shihai, Jin Fenghua. Research on Dynamic Elevation Measurement System of Turbine Generator Set Bearing Based on LabVIEW[J]. Power Station System Engineering, 2018, 34(5): 5-8.

[7] Xueshen, Ye Xining. Distributed control system and its application[M] Beijing: Mechanical Industry Press. 2015(2): 137-142.

[8] Zhou Hong,Wang Leyu,Chen Xiangxian.The software structure design of virtual instrument system[J].Computer Automatic Measurement and Control ,2000,4(20):20-40.

[9] MARTINS HA,BIRK JR,KELLEY RB. Camera models based no data from two calibration planes[J]. Computer Graphics and Imaging Processing, 1981, 17 ( 2 ):173- 80.

[10] Dong Yu,Ding Jinhua,Shen Jianfeng.Real-time analysis system of vibration test based on LabVIEW[J]. Instrument Technology and Sensor, 2012(11): 47-49.

[11] Yang Xiufang. Zhang Xining. Research on Analysis and Extraction Method of Vibration Signal of Gear Partial Failure[J]. Journal of Xi'an University of Technology, 2019. 35(1): 28-33.

[12] Tang Guiji, Ma Wanli, Dai Tuo, et al. Design and application of a virtual vibration test and analysis system based on Lab VIEW[J]. Instrument Technology and Sensors, 2011(11): 54-56, 59.

[13] Tang Yi, Li Qi, Wang Wenjuan. Design of Vibration Signal Analysis System Based on LabVIEW[J]. Computer Measurement and Control, 2016, 6( 60) 218-222. 